\title{
The Research on the $180^{\circ}$ Upender Motion and Mechanics Of Nuclear Large Pieces
}

\author{
Dengyue Sun, Peng Chen, Shiming Xu and Hailong Li \\ ( National Cold Rolled Strip Equipment and Process Engineering Technology Research Center, \\ Yanshan University ,Hebei Qinhuangdao 066004 , China )
}

Keyword: upender, nuclear power ,large pieces, motion, mechanics, reliability analysis.

\begin{abstract}
The attitude displacement process of nuclear power large piecesis time-consuming and very dangerous, which has became one of the important factors for improving productivity and enhancing the workpiece's surface quality.However, the research and development of upenders for nuclear power large parts is seldom reported no matter in domestic or abroad. The method that crown with wire ropeis commonly used, which is very low controllability. It carries out the research based on a nuclear large pieces $180^{\circ}$ upender which we jointly developed with a heavy machinery factory. With the application of theoretical analysis and simulation analysis, the complex stressstate of dangerous sport nodes and the turning process in the equipment's operation process that upender completes the most difficult and most dangerous 300 tons dome head 180-degree shift is analyzed. The study result ensures the safety and reliability of the equipment for trial operation. It provides reliable theoretical for equipment structure optimization, dynamic parameter selection. There search also plays an important reference role for making turning process of different kinds of nuclear power large pieces.
\end{abstract}

\section{Introduction}

Recently years, with fossil fuel prices higher and the impact of global climate deterioration, many countriesre-examinethe strategystatus of nuclear power in the national energy security andagree that the development of clean and economy nuclear power is necessary to achieve economic sustainable development ${ }^{[1,2]}$. At present, many heavy machinery group companies introduce and absorb foreign advanced nuclear power technology and have mastered completely multifaceted nuclear power equipment manufacturing core technology, including pressure reactor, steam generator, voltage regulator, etc. The above equipment is composed of a number of the tapered transitional section, the dome head, the special thick pie and the big drum section forgings bywelding ${ }^{[3,4]}$. They have a common feature that size huge, super quality, complex structure. The attitude displacement process of nuclear power large pieces is time-consuming and very dangerous, which has became one of the important factors for improving productivity and enhancing the pieces surface quality.

At present, the method of crown with double wire rope is widely used toachieve large parts turnover in the domestic as shown in Figure1. The working process is that the upper driving and lower driving liftpieces at the same time to make it roll over on two ropes. The method described above has some drawbacks, such as poor process control ,piecessurface damage andscrapped, even it will cause injury accident. It is of great significance to research and develop an nuclear power large pieces turnover deviceovercoming the traditional flip process faults ${ }^{[5,6]}$.

Facing with the difficult situation of large pieces flip and displacement ${ }^{[7,8]}$, We spend millions to research and design a device that can make many types of complex large heavy pieces achieve 90 / 180 degree flip and displacement with high efficiency. Currently, the device is in the final assembly phase, three-dimensional virtual prototype and equipment test site is shown in Figure 2.

Involved in the design process of upender, we found the mechanical state ofeachkey motion node is complex and dynamic changes in the process of pieces deflection.It has important reference valueto obtain dynamic load changes in the value of key nodesunder the ultimate loadfor important 
dynamic parameter selection, structure optimization, safe operation of the equipment.
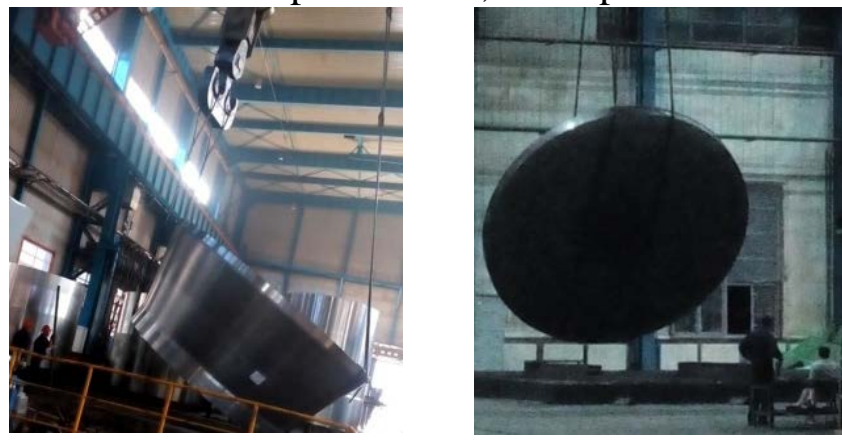

Fig.1The scene of nuclear large pieces turning
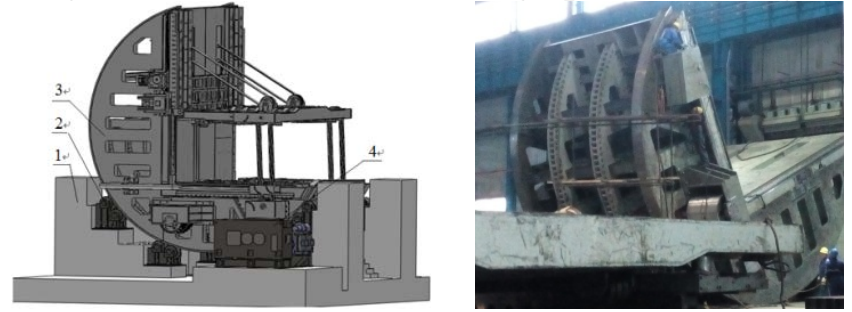

1-L-type body2-Mobile and flip platforms

3-Chain drive system4-Support base

Fig.2The basic structure of upender

\section{Working principle of the upender}

The process of upender achieving the pieces180 degree flip and displacement is very complicated $^{[9]}$. Takingthe most difficult and most dangerous flip and displacement of limit of 300 tons piecesfor example, We analyze the working process of upender.The mobile workbench and the flip table are stacked together in the initial position, The cranehoists pieces to L-type table.Flip table is rotated90 degrees under the positive and reverse winch system coordination control,forming a u-shaped structure with L-shaped bench, which realizes the axial and radial of the pieces fixation.The main body is rotated more than 90 degrees to b-location so that the center of gravity of the pieces is shifted the other side of the hinge pin of mobile bench.The center of gravity of the pieces falls on turning table.The flip angle ofdifferent specifications pieces in the b-station is variable.The analysis and calculation of variable value is necessary.Thenthe turning table carrying pieces rotates around the hinge pin of turning table under the positive and reverse winch system coordination control and L-shaped bench rotates reversely meanwhile until the two bench stacking together as shown in Figure 3. The purpose of e-location is to improvethe stress state of key sports node. The flip angle ofdifferent specifications pieces in the e-station is variable.Finally the upender and pieces are rotated to a horizontal position to achieve the pieces 180 degree flip and displacement $^{[10]}$.

\section{Technology research on variable-position}

The flip angle of different specifications pieces in the b\&c-station is variable when the upender achievesthe pieces 180 degrees flip and displacement, which relates to the equipment safety

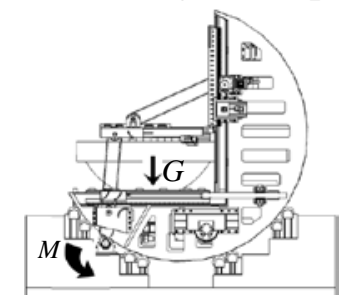

a-location

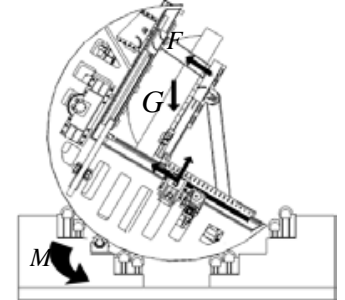

b-location

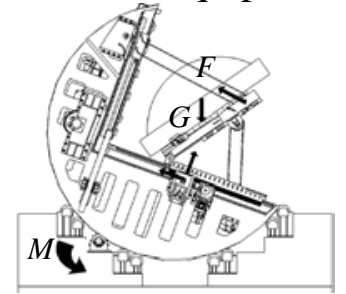

c-location 


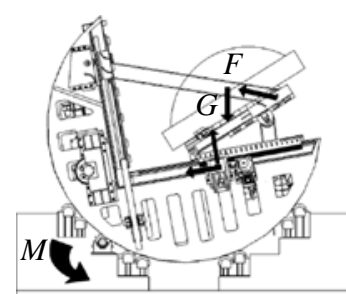

d-location

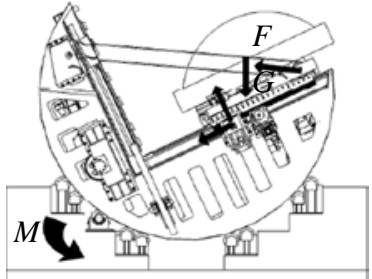

e-location

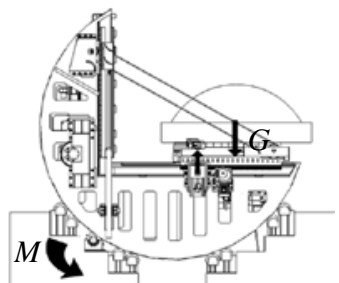

f-location

Fig.3 The motion process of upender

and efficient operation. The purpose of b-location is to shift the center of gravity of the pieces to the other side of the hinge pin of mobile bench insuringthe center of gravity of the pieces falling onmobile bench. The purpose of e-location is to improve the stress state of the key sports node insuring the equipment safety.we research the best flip technology of the variable-positionbased onthe most difficult and most dangerous flip and displacement of limit of 300 tons pieces, which providesa reference analysis method for the best flip technology of other special type of pieces $^{[11]}$.

\subsection{Technology research on b-position}

The trajectory of pieces center of gravity A and the pin axis center $G$ is shown in Figure 4 in the position movement process from a to $b$.

In order to analysis and calculate conveniently, we set $O(0,0)$ as the center coordinate of rotational body.The coordinates of the pieces gravity center $\mathrm{A}$ is $\left(1408-l_{A F},-1187\right)$. The coordinates of the hinge pin center $\mathrm{G}$ of mobile and flip table is $(1110,170)$. We need to guarantee that the pieces center of gravity $A$ is greater than the center of the hinge pin $G$ in b-location so that the center of gravity of the pieces is shifted to mobile bench.The relative position of the pieces and the upender doesn ' $t$ change in the movement process. The angular size of $\theta$ is constant. The relationship is got based on the above analysis:

We get the analytical value of 27.97 degrees. So the Critical turning Angle is $117.97^{\circ}$ when the center of gravity of the pieces is shifted to the other side of the hinge pin of mobile bench in b-location. Restricted by upender structure and the chain transmission system design,the limit rotation angle is $123^{\circ}$ from a- location to b- location. Based on the above analysis,The optimal flip angle of the pieces is set to $120^{\circ}$.

\subsection{Technology research on e-position}

The purpose of e-location is to improve the stress state of the key sports node insuring the equipment safety,However the unreasonable rotation angle will reduce the efficiency of the equipment and increase energy consumption.Making the best reverse process on e-location, We should take into account the intensity and efficiency of equipment.The wire rope and pulley are subjected to complex mechanical conditions. It is the most easy to be destroyed when the upender

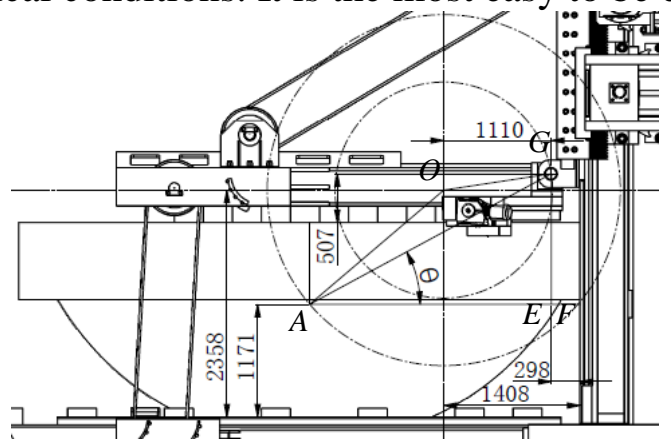

Fig.4 The trajectory of head gravity andpincenter

$$
\begin{gathered}
\tan \theta=\frac{l_{G F}}{l_{A E}} \\
l_{A F}=l_{1} \tan \gamma-\frac{R}{\cos \gamma}-l_{2}
\end{gathered}
$$




$$
\theta=\arctan \frac{1357 \cos \gamma}{l_{1} \sin \gamma-\left(l_{2}+298\right) \cos \gamma-R}
$$

$R$ - Piece radius of big end.

$\gamma$-Bottom edge angle of the V-type groove .

$\mathrm{l}_{2}$ —Depth values of V-groove .

$l_{1}$ _- Half value V-groove bottom width.

achieves the limit pieces of 300 tons 180 degree flip and displacement.The basic research idea of turning process on e-location is to analysis the maximum allowable tension of the wire rope and pulley and calculate the safety turningangle on e-station ${ }^{[12]}$.

The main influencing factors of the allowable tension of the wire rope and pulley includes compressive strengthand shear strength of pulley shaft, tensile strength of ropeand so on.The material of pulley shaft is $40 \mathrm{Cr}$ and $100 \mathrm{~mm}$ indiameter.By heat treatment, the yield strength of the shaft reaches 870MPa.The design safety factor of the upender is 2. For plastic materials, the equation of shear allowable stress and squeeze allowable stress and tensile allowable stress is as follows:

$$
\begin{array}{r}
{[\tau]=(0.6 \sim 0.8)[\sigma]} \\
{\left[\sigma_{b s}\right]=(1.7 \sim 2.0)[\tau]}
\end{array}
$$

The equation of wire rope allowable tension:

$$
\begin{gathered}
{[F] \leq 4 F_{Q}=4 \pi R^{2}[\tau]} \\
{[F] \leq 10 F_{b s}=10 A_{b s}\left[\sigma_{b s}\right]}
\end{gathered}
$$

Based on analysis of shear strength, thesafety allowable tension of the wire rope and pulley block is $10932.7 \mathrm{kN}$; Based on analysis of crushing strength, thesafety allowable tension of the wire rope and pulley block is $59160 \mathrm{kN}$.The minimum breaking force of single wire rope is $298 \mathrm{kN}$.The maximum force of wire rope acting on the pulley block is $5960 \mathrm{kN}$.Considering of safety factor of steel wire rope andthe above analysis, thesafety allowable tension of the wire rope and pulley block is $1192 \mathrm{kN}$.

The mechanics analysis of upender on e-location is shown in Figure 5;

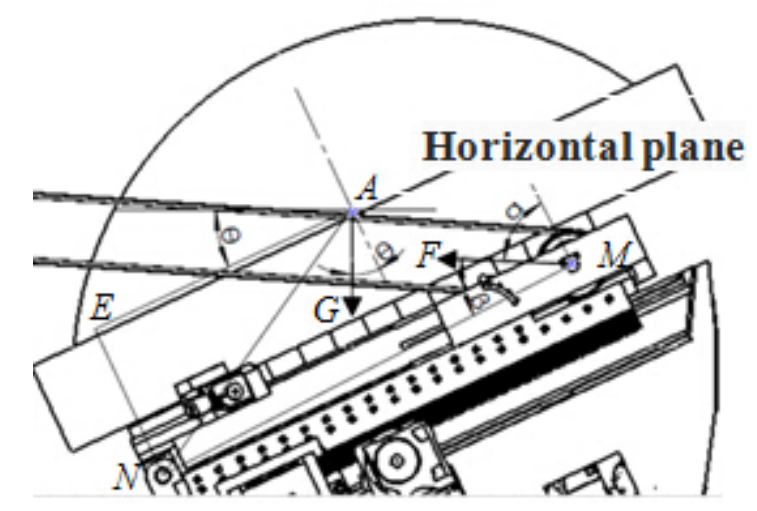

Fig.5 The body of upender mechanics analysis

The relationship of wire rope tension:

$$
\begin{gathered}
F=\frac{\left(2 G l_{A E}+G_{1} l_{N M}\right) \cos \theta-2 G l_{N E} \sin \theta}{2 l_{N M} \cos \alpha} \\
l_{A E}=l_{1} \tan \gamma-\frac{R}{\cos \gamma}-l_{2}-298 \\
\alpha=90^{\circ}-\arctan \frac{l_{P Q}}{l_{M Q}}
\end{gathered}
$$

G_-Gravity of pieces.

$G_{1} \_$Gravity of overturning platform.

$l_{A E}$-Distance between center of gravity with the vertical of the hinge. 
$l_{N E}$ Distance between center of gravity with the plane of the hinge.

$l_{N M}$ —Distance between the pulley center with thehinge center.

$l_{\text {MQ }}$ Distance between the pulley of flip platformwith the platform.

$l_{P Q}$ Distance between the pulley ofthe body with the platform.

We get the angle relationship between body and the ground by above analytic and the maximum allowable tension of the pulley:

$$
\begin{gathered}
\theta=-\arcsin \frac{2[F] l_{N M} \cos \alpha}{\sqrt{\left(-2 G l_{N E}\right)^{2}+\left(2 G l_{A E}+G_{1} l_{N M}\right)^{2}}} \\
-\arctan \frac{\left(2 G l_{A E}+G_{1} l_{N M}\right)}{-2 G l_{N E}}
\end{gathered}
$$

$[F]$ — the maximum allowable tension of the pulley.

Considering the above analysis and structure size of equipment, We obtain that the best flip angle of limit pieces is $49.3^{\circ}$ in e-location.

\section{Conclusion}

(1) This paper proposes a novel upender scheme which can realize a $90^{\circ} / 180^{\circ}$ turning of nuclear large pieces. Itsolvesthe dangerous and difficult situation that the displacement of large pieces. It has a certain reference value for the design research and application promotion of upender.

(2)The best flip solution of flipping the extreme 300 tons piece is $120^{\circ}$ in b-location and $49.3^{\circ}$

in e-location. The proposed method and dynamics equation are still effective for the reliability analysis and the formation of the best flip technology when other special types of pieces are flipped by the upender.

\section{References:}

[1] Wang Yuan-long.Localization of nuclear power equipment in China[J]. Science \& Technology Review,2012, 30(20): 65 70.

[2] XiaoJian-xin.Chinese nuclear power development situation in 2011, future trend and policy suggestion.Energy of China, , 2012, 34 (2) : 18 23.

[3] Gao F. Artificial Intelligence and Robotics in Manufacturing[M]. CRC Press LLC,USA,2011.

[4] Lee K M, Shah D K. Dynamic analysis of a three-degrees-of-freedom in-parallel actuated manipulator[J]. Robotics and Automation, IEEE Journal of, 1988, 4(3): 361-367.

[5] Carricato M, Parenti-Castelli V. A Novel Fully Decoupled Two-Degree-of-Freedom Parallel Wrist[J]. The International Journal of Robotics Research, 2004, 23 (6):661 667.

[6] Van J, StackleyM. Lowering Upender Design and Synithesis[J].Biennial Mechanisms and Robotics Conference,2004,2(28):709-716.

[7] Huang Lin.Design of turnover machine for flat truck[D]Tian Jin, TianjinUniversity,2012;4-8.

[8] XieDongdong.The design and research of Hydraulic and pneumatic system for 22t/h Aluminum ingot Turnover Mechanism[D]. Lanzhou. Lanzhou University of Technology.2011;1-4.

[9] Zhou Yu-lin, Zhao Yong-sheng, QiuXue-song. Three freedom degrees $90^{\circ} / 180^{\circ}$ Upender[P]. China, 201210243957.X,2012-7-16.

[10]Xue Song-qiu, Liang Wang,Lei Wang. Development of a 90\% $180^{\circ}$ Upender for Large and HeavyWorkp-iece by Usi-ng Airbags-Drived Flipping Workta ble[J].Advanced Materials Research, 2014,3140( 915): 228 235.

[11]Zhang Zhen-bo, Ma Yan-hong, Li Jun, etal. Research onthe dynamic modle of 
aero-engingrotorswithbearingmisalignment[J]. Engineering Mechanics, 2013: 31(7). 208-212.

[12] ShenHui-ping, GaoZong-hua, Qi Ze-pei. Turnover mechanism of the turning mechanism of tunnel segment vacuumsucker tipper and its optimal calculation of minimum turning torque[J],Journal of Machine Design,2010,27(5):63 66+85. 